\title{
RASTREIO DO CÂNCER DE COLO UTERINO NO ESTADO DO PARANÁ: INDAGAÇÕES SOBRE A FAIXA ETÁRIA PRECONIZADA
}

\author{
SCREENING FOR CERVICAL CANCER IN THE STATE OF PARANA: \\ INQUIRIES ABOUT THE RECOMMENDED AGE RANGE
}

Vitória Dolci Rodrigues*, Kamila Bolotário, Nathália de Carvalho Balan, Raphaela Montalvão Morais, Taís Lorrane Mendes Silva, Vanessa Sarto Soares Bergamasco.

UNINGÁ - Centro Universitário Ingá, Maringá, PR, Brasil. *vitoriadr@hotmail.com

\section{RESUMO}

Desde 1998 o Brasil implantou o Programa Nacional de Controle do Câncer de Colo para mulheres entre 25 a 64 anos para aprimorar o diagnóstico do câncer cervical uterino e reduzir o índice de mortalidade pela doença. $O$ rastreamento do câncer cervical do útero é realizado pelo exame citopatológico do colo do útero (teste Papanicolau), disponível nas unidades básicas de saúde do Sistema Único de Saúde e na rede privada. A idade de início do rastreamento tem sido questionada, já que a mulher tem iniciado sua vida sexual mais cedo, em média com 14,9 anos. Além disso, é importante avaliar se pacientes entre 20 a 29 anos diagnosticadas com carcinoma de colo uterino apresentam progressão mais rápida, analisando fatores clínicos, critérios anatomopatológicos, desenvolvimento da doença e expressão de biomarcadores. Este estudo visa analisar se existe a necessidade de realizar rastreamento na faixa etária abaixo dos 25 anos, devido à sexarca mais precoce e à exposição ao vírus do HPV, com base nos dados epidemiológicos no período de 2018 a 2019 no Estado do Paraná. Os bancos de dados utilizados foram o Departamento de Informática do Sistema Único de Saúde (DATASUS) por meio do Sistema de Informação do Câncer (SISCAN). A população em estudo corresponde a mulheres com faixa etária entre 20 e 39 anos do estado do Paraná, no período de janeiro de 2018 a dezembro de 2019. Achados dos resultados mostram que foram realizados 514.598 exames de rastreamento para câncer de colo uterino entre 2018 e 2019 e desses, 108.730 (21.2\%) exames estavam com resultado alterado. Na faixa etária entre 20 a 24 anos, dos 119.427 exames, 21.904 (18,34\%) estavam alterados. Desses resultados alterados, 10 ou $0.04 \%$ das mulheres tinham câncer e 2 delas foram à óbito. Na faixa etária entre 25 a 30 anos, foram realizados 126.073 exames, desses 25.553 (20.26\%) estavam alterados. Desses resultados de exame alterados, $23(0.09 \%)$ eram câncer de colo uterino e 6 foram à óbito. Na faixa etária entre 30 a 34 anos realizou-se 128.563 e 28.137 $(21.88 \%)$ resultados estavam alterados. Desses, $54(0.19 \%)$ eram câncer de colo e 22 pacientes foram à óbito. Na faixa etária entre 35 a 39 anos foram realizados 140.535 exames e desses, $33.136(23.57 \%)$ tinham resultado alterado. Dos resultados alterados, $74(0.22 \%)$ eram câncer de colo uterino, com 30 óbitos devido a essa patologia. A média das porcentagens de exames alterados em relação ao total de exames realizados em cada faixa etária é de $21.02 \%$, com 
desvio padrão de 2.68. Conclui-se que a incidência de exames de rastreio para câncer de colo uterino com resultados alterados é de 21\% (DP 2,68\%). Entre 20 a 24 anos é necessária coleta de muitos exames para evitar um pequeno número de óbitos, diferente das outras faixas etárias. Esses dados reforçam os estudos já existente na literatura, que mostram a lenta evolução das lesões precursoras relacionadas ao HPV na abaixo dos 25 anos e alta taxa de regressão espontânea da mesma.

Palavras-chave: Câncer de colo uterino. Gammapapillomavirus. Ginecologia. Rastreamento. Teste de Papanicolaou. 